\title{
Landwirtschaft und Raumordnung im ländlichen Raum (Schweizerisches Mittelland)
}

\section{Raumplanung im ländlichen Raum}

Spricht man vom ländlichen Raum, so denkt man an einen Raum, in dem dörfliche Siedlungen, Wiesen, Äcker, Wälder, Flüsse, Bäche und Seen miteinander abwechseln - an einen durch Landwirtschaft und Forstwirtschaft geprägten Raum. Ohne hier auf eine formale resp. funktionale Abgrenzung des ländlichen Raumes einzutreten, stellt sich für das Schweizerische Mittelland die Frage, ob für dieses noch ländliche Räume mit Siedlungsschwerpunkten abwechseln oder ob es eher als Ganzes gesehen als verstädterter Raum zu behandeln sei. Wie auch immer je nach Standpunkt die Antwort ausfallen wird, so verdeutlicht diese Frage die gegenwärtige Situation in bezug auf die Nutzungsansprüche im Schweizerischen Mittelland. Insbesondere während des Baubooms in den sechziger und anfangs der siebziger Jahre sind die Siedlungen in das Land hinausgewuchert. Infrastrukturanlagen wie die Erstellung des Nationalstraßennetzes, die Anlage von Flughäfen, die Erstellung von Kläranlagen, die Anlage von Deponien, der Abbau von Steinen und Erden usw. beanspruchten und beanspruchen immer mehr Kulturland. Einhergehend mit dieser Bautätigkeit war es notwendig, Flüsse und Bäche zu korrigieren. Alle diese Entwicklungen sind in bezug auf den Verbrauch an gewachsenem Boden zulasten der Landwirtschaft gegangen, da durch das Forstpolizeigesetz der Wald in seinem Bestand geschützt ist.

Eindrücklich belegen Untersuchungen diese Tatsache, wonach in der Schweiz ca. $1 \mathrm{~m}^{2}$ Kulturland pro Sekunde verloren geht (HÄBERLl und STADLER 1979). In bezug auf die Gesamtschweiz konzentriert sich dieser Verlust an gewachsenem Boden vornehmlich auf das Mittelland. Dabei bezieht sich der Verlust keineswegs auf die ökologisch wenig wertvollen Flächen, sondern es sind gerade jene Böden, die sich als ökologisch besonders reich ausweisen, also Böden mit einem vielfältigen Naturpotential (1). Dies ist teilweise historisch begründet, indem Siedlungsschwerpunkte gerade inmitten wertvoller landwirtschaftlicher Gebiete oder an See-Enden und entlang von Flußläufen entstanden sind, da damals Gewerbe, Industrie und Siedlungstätigkeit viel unmittelbarer von der ökologischen Standortgunst abhängig waren. Diese Zusammenhänge hat KAULE (1980) sehr eindrücklich für das untere und mittlere Neckartal und den Bodenseeraum in Baden-Württemberg nachgewiesen. Es kommt daher auch nicht von ungefähr, daß $3 / 4$ der Einwohner der Schweiz sich auf $1 / 4$ der Fläche, dem Mittelland, konzentrieren.

Es sind aber nicht nur Siedlungsentwicklung und der Bau von Infrastrukturanlagen, die das Schweizerische Mittelland umgestaltet haben, sondern auch die Landwirtschaft hat maßgebend $z u$ einem neuen Gesicht der Landschaft beigetragen. Mit der Entwicklung der Agrochemie und der Technisierung der Landwirtschaft, insbesondere der Mechanisierung, und unter dem Zwang, möglichst rationell zu wirtschaften, haben sich die Produktionsstrukturen in der Landwirtschaft tiefgreifend verändert. Insbesondere ist hier die Gesamtmelioration als Strukturverbesserungsmaßnahme zu nennen. Wie ausgeprägt die Umgestaltung der Landschaft durch die Veränderung der landwirtschaftlichen Produktionsstrukturen war und ist, zeigt mit aller Deutlichkeit das Buch von EWALD (1978).

Als Auswirkungen und z. T. als Reaktion auf die geschilderten Entwicklungen ist der erhöhte Erholungsdruck im ländlichen Raum und die Forderung nach Einrichtung von Landschafts- und Naturschutzgebieten $\mathrm{zu}$ verstehen.

Zusammenfassend läßt sich festhalten, daß der ländliche Raum mannigfachen Nutzungsinteressen, die sich zum Teil gegenseitig ausschließen, überlagern, beeinträchtigen oder unterstützen, ausgesetzt ist. Zudem konzentrieren sich die Interessen vorwiegend auf die ökologisch wertvollen Gebiete, mit den somit höchsten Nutzungspotentialen. Dies gilt im übrigen nicht allein für das Schweizerische Mittelland, sondern ebenso für das Berggebiet, wo eine Häufung der Nutzungsansprüche in bevorzugten Tallagen festzustellen ist.

Ließe man nun alle Interessenten an Grund und Boden frei gewähren, so würde sich der vor allem wirtschaftlich Stärkere durchsetzen, und das Resultat wäre eine ungeordnete Raumbeanspruchung, was, wie die vergangene räumliche Entwicklung zeigt, zu katastrophalen Folgen führen muß.

Willy A.Schmid, Prof. Dr., Institut für Orts-, Regional- und Landesplanung, ETH-Hönggerberg, 8093 Zürich 
Basierend auf der Einsicht, daß der Boden keine vermehrbare Ressource darstellt und dieser daher haushälterisch zu nutzen sei, wird Raumplanung als Aufgabe des Staates zur Wahrung öffentlicher Interessen gesehen, die zu Eigentumsbeschränkungen führen kann (2). Die Hauptaufgabe der Raumplanung besteht demnach darin, räumliche Konflikte zu bewältigen und räumliche Ordnung zu schaffen. Wenn in den sechziger Jahren die Raumplanung vor allem identisch mit Siedlungsplanung war, so erfaßt sie heute den Gesamtraum und insbesondere auch den ländlichen Raum, indem sie gemäß Raumplanungsgesetz im Nichtsiedlungsgebiet zumindest zwei Zonen - Landwirtschaftszonen und Schutzzonen auszuscheiden hat. Das zentrale Anliegen der Raumplanung ist nach wie vor die klare Trennung des Nicht-Siedlungsgebietes vom Siedlungsgebiet. Die Raumplanung weist den jeweiligen Flächen Nutzungen zu und dimensioniert diese, indem sie den zulässigen oberen Grenzwert der Nutzung bezeichnet. Allerdings ist bis heute die Raumplanung im ländlichen Raum zu sehr flächenorientiert. D.h., man konzentriert sich beinahe ausschließlich darauf, Nutzungsinteressen zu entflechten und diese einzelnen Flächen zuzuordnen; eingeschlossen sind dabei Landschafts- und Naturschutz, wobei sich durchaus Nutzungsüberlagerungen ergeben können. Kaum einbezogen werden dabei die Wirkungen der Nutzungen auf den Landschaftshaushalt insgesamt. Eine flächenmäßige Zuordnung der Nutzungen, basierend auf der gegenseitigen Abwägung einzelner Nutzungsinteressen und ästhetischer Gesichtspunkte, kann aber nicht genügen, wie z.B. das Waldsterben zeigt, das durch die flächenmäßige Bestandesgarantie des Forstpolizeigesetzes nicht verhindert werden konnte. Es ist daher zu fordern, daß die Frage nach den ökologischen Auswirkungen von Nutzungszuweisungen mit in die raumplanerischen Überlegungen einbezogen wird. Unter anderem handelt es sich hier um das Verhältnis zwischen Raumplanung und Umweltschutz, wobei die Umweltplanung selbst wie die Raumplanung eine querschnittsbezogene, teilintegrierende Planung darstellt. Die Raumplanung tut sich daher und wegen eines in bezug auf das Vorgehen fehlenden methodischen Konzeptes schwer, Umweltschutz resp. ökologische Forderungen in den Planungsprozeß zu integrieren. Es wäre sicher falsch, wenn die Raumplanung Forderungen des Umweltschutzes als Randbedingung der Planung verstehen würde; ihre Aufgabe besteht vielmehr darin, diese in ihrem Raumbezug im Raumplanungsprozeß zu verarbeiten (3).

Auf der einen Seite ist es das Ziel der Raumplanung, einen zweckmäßigen und sinnvollen Ausgleich der Nutzungsinteressen anzustreben, der einerseits den Erhalt der Landschaft gewährleistet und andererseits das wirtschaftliche Gedeihen des Landes nicht behindert oder gar verhindert. Auf der andern Seite sieht sich die Landwirtschaft einer Vielzahl von nicht landwirtschaftlichen Nutzungsansprüchen ausgesetzt, ausgehend vom Siedlungsdruck, der Inanspruchnahme von Land für Infrastrukturvorhaben über den Kiesabbau bis hin zum Natur- und Landschaftsschutz. Um keine Mißverständnisse aufkommen zu lassen: hier wird von den landwirtschaftlichen Interessen gesprochen und nicht vom Grundeigentümer, der landwirtschaftliches Land besitzt und durchaus gleichzeitig verschiedene Nutzungsinteressen haben kann.

Es stellt sich somit die Frage: In welchem Verhältnis stehen landwirtschaftliche Planung und Raumplanung zueinander? Diese Frage läßt sich dem Spannungsfeld zwischen sektoraler Politik wie Wirtschaftspolitik, Bildungspolitik, Forstpolitik, Landwirtschaftspolitik und der querschnittsbezogenen Raumordnungspolitik zuordnen (LENDI 1984). Allerdings ist der Landwirtschaft insofern eine Sonderstellung gegenüber anderen Nutzungen einzuräumen, indem sie aus der Defensive heraus ihre Ansprüche durchsetzen muß.

\section{Landwirtschaftliche Planung und Raumplanung}

Entsprechend den agrarpolitischen Zielsetzungen, hat sich die landwirtschaftliche Planung auf die Erhaltung eines gesunden Bauernstandes, Versorgung des Landes mit Nahrungsmitteln in Normalzeiten und bei gestörter Zufuhr und die Erhaltung der Landschaft auszurichten. Damit sieht sich die landwirtschaftliche Planung mit folgenden Fragen konfrontiert:

- Wie ist eine ausreichende Versorgungsbasis sicherzustellen, wobei man in Normalzeiten von der Produktionsplanung und in Zeiten gestörter Zufuhr vom Ernährungsplan spricht.

- Welche Böden in quantitativer und qualitativer Hinsicht sind daher landwirtschaftlichen Zwecken zu erhalten?

- Wie sind Konflikte zwischen der Erhaltung von Bauernbetrieben und der Siedlungsentwicklung (Bauernhöfe in der Bauzone, Rückzonung von Baugebieten) und weiteren Nutzungen, insbesondere Natur- und Landschaftsschutz, zu lösen?

Angesichts solcher Fragen wird deutlich, daß im Zusammenhang mit der Raumplanung der landwirtschaftlichen Planung in erster Linie die Aufgabe zukommt, die Interessen der Landwirtschaft in die Raumplanung einzubringen. Umgekehrt ist aber Raumplanung und Raumordnungspolitik zielbestimmend für die landwirtschaftliche Planung. Die landwirtschaftliche Planung ist demnach eine von Agronomen, Agrarökonomen, Kulturingenieuren usw. durchzuführende eigenständige Planung.

Die Raumplanung selbst kann diese Aufgabe nicht übernehmen. Hingegen hat die Raumplanung die landwirtschaftliche Planung in ihrem Raumbezug zu erfassen. Es ist offenbar, daß dieser Raumbezug für die Landwirtschaft äußerst ausgeprägt ist, so daß jede agrarpolitische Entscheidung auch raumplanerisch relevant ist. Die Landwirtschaft erhält somit im Rahmen der nominellen Instrumente der Raumpla- 
nung, der Richtplanung und der Nutzungsplanung eine vorrangige Bedeutung, und es muß daher auch ein zentrales Anliegen der Raumplanung sein, daß die Landwirtschaft ihre Anliegen entsprechend im Rahmen des Raumplanungsprozesses vertritt.

Ausgehend von diesen Überlegungen, sind in der Richtplanung, welche in ihrer Funktion die zukünftige Raumstruktur aufzeigen soll und vor allem der Koordination der raumwirksamen Tätigkeiten dient, in bezug auf die Landwirtschaft zunächst die landwirtschaftlich geeigneten Böden auszuweisen, und zwar hinsichtlich einer ausreichenden Versorgungsbasis für die Schweiz mit Nahrungsmitteln. D. h., es sind in der Raumplanung folgende Gebiete aufzuzeigen:

- landwirtschaftliche Vorrangflächen

- Flächen, deren landwirtschaftliche Nutzung im Gesamtinteresse stehen

- Richtflächen

- Fruchtfolgeflächen

- Flächen, wo Konflikte zwischen landwirtschaftlichen Vorrangflächen und nicht landwirtschaftlichen Nutzungsansprüchen bestehen (z. B. landwirtschaftlich genutzte Bauzonen, Erholungsgebiete, touristische Gebiete, Schutzgebiete).

Als landwirtschaftliche Vorrangflächen sind jenes Wies- und Ackerland und jene Flächen für Reb-, Obst- und Gartenbau zu bezeichnen, deren natürliche Ertragsfähigkeit im regionalen Vergleich hoch ist und die maschinell bewirtschaftbar sind sowie vorwiegend in ganzjährig besiedelten Gebieten liegen.

Unter jenem Land, das im Gesamtinteresse landwirtschaftlich zu nutzen ist, werden jene Gebiete verstanden, die zwar nicht zu den landwirtschaftlichen Vorrangflächen zu zählen sind, aber aus Gründen der Erhaltung der Dauerbesiedlung, des Schutzes der Landschaft oder der ergänzenden Landesversorgung land- und alpwirtschaftlich genutzt werden sollen.

Richtflächen sind ackerbaulich nutzbare Flächen, die in Zeiten drohender Störung der Zufuhr durch den Bundesrat mit zeitlich beschränkten Anbauverpflichtungen belegt werden können. Sie betragen zurzeit gesamtschweizerisch 290000 ha und werden durch den Bund auf die Kantone und von diesen auf die Gemeinden und einzelnen landwirtschaftlichen Betriebe verteilt. Die Richtflächen dienen agrarpolitischen Zwecken und bilden die Vorstufe zur Ernährungsplanung.

Im Falle einer Versorgungskrise muß die offene Ackerfläche von heute rund 278000 ha in drei Mehranbaustufen auf 355000 ha erhöht werden. Da die für die Basisernährung notwendigen Kulturen wie Brotgetreide, Raps, Kartoffeln in Rotation mit Kunstwiesen anzubauen sind, werden gesamtschweizerisch im Minimum 450000 ha ackerfähiges Kulturland, die als Fruchtfolgeflächen bezeichnet werden, benötigt.

Diese Flächen verteilen sich wie folgt auf die Kantone:

Minimale Fruchtfolgeflächen (Ackerland + Kunstwiesen in Rotation) zur Sicherung der ausreichenden Versorgungsbasis, ohne Kleinpflanzerflächen, für $\mathrm{KS} 3^{\star}$, in Hektaren

\begin{tabular}{|c|c|c|c|c|c|}
\hline Zürich & 40500 & Fribourg & 38500 & Aargau & 40000 \\
\hline Bern & 90800 & Solothurn & 18400 & Thurgau & 26000 \\
\hline Luzern & 26500 & Basel-Stadt & 260 & Ticino & 4520 \\
\hline Uri & 200 & Basel-Land & 9460 & Vaud & 74000 \\
\hline Schwyz & 1760 & Schaffhausen & 9700 & Valais & 7500 \\
\hline Obwalden & 470 & Appenzell A.-Rh. & 800 & Neuchâtel & 8200 \\
\hline Nidwalden & 360 & Appenzell I.-Rh. & 360 & Genève & 10500 \\
\hline Glarus & 360 & St. Gallen & 15850 & Jura & 14800 \\
\hline Zug & 2700 & Graubünden & 7500 & TOTAL & 450000 \\
\hline
\end{tabular}

aus: EJPD/BRP; Grundlagen, Konzepte, Sachpläne und Bauvorhaben des Bundes (Übersicht); Teil BLW

Es ist evident, daß Richtflächen zugleich auch Fruchtfolgeflächen sind. Selbstverständlich sind Fruchtfolgeflächen zugleich auch landwirtschaftliche Vorranggebiete, während landwirtschaftliche Vorranggebiete nicht zugleich Fruchtfolgeflächen sein müssen (ARBEITSGRUPPE "RAUMPLANUNG UND LANDWIRTSCHAFT DES SCHWEIZERISCHEN BAUERNVERBANDES", 1983).

Gemäß Raumplanungsgesetz ist in der Nutzungsplanung die grundeigentumsverbindliche Ausscheidung von Landwirtschaftszonen zwingend vorgeschrieben.
Es liegt auf der Hand, daß grundsätzlich alle landwirtschaftlichen Vorranggebiete der Landwirtschaftszone zuzuordnen sind. Überall dort, wo Konflikte zwischen Fruchtfolgeflächen resp. landwirtschaftlichen Vorranggebieten im Berggebiet und nicht landwirtschaftlichen Nutzungen auftreten, ist in der Regel der Konflikt zugunsten der Landwirtschaft zu lösen. Ausnahmen sollen möglich sein. In allen übrigen Fällen wird es von den regionalen und lokalen Verhältnissen abhängen, wie die Konfliktlösung zu erfolgen hat. 
Mit der Einrichtung der Landwirtschaftszonen ist die Nutzungsplanung für die Landwirtschaft keineswegs abgeschlossen, sondern es ist in der Folge dafür zu sorgen, daß die der Landwirtschaft zugeordneten Flächen auch entsprechend genutzt werden können. Dazu sind in vielen Fällen entsprechende Strukturanpassungen vorzunehmen. Eine der wichtigsten agrarpolitischen Strukturverbesserungsmaßnahmen stellt die Güterzusammenlegung als Parzellarordnungsmaßnahme mit entsprechender Infrastruktur, vor allem Wegebau und weiteren Maßnahmen wie landwirtschaftlicher Hochbau, Entwässerungen und Bewässerungen dar. Konsequenterweise ließe sich die Ansicht vertreten, daß sich die Güterzusammenlegung auf die als Vorranggebiete ausgeschiedenen Flächen beschränken soll. Zugegeben, dies würde die Durchführung von Zusammenlegungen sicher erleichtern, da in solchen Gebieten die landwirtschaftlichen Interessen bei weitem überwiegen, und es besteht durchaus verschiedentlich die Auffassung, Güterzusammenlegungen auf unbestrittene Landwirtschaftsgebiete $\mathrm{zu}$ begrenzen.

Was geschieht jedoch in diesem Falle mit all jenen Flächen, die zwar heute noch landwirtschaftlich genutzt werden, aber längerfristig durch andere Nutzungsarten beansprucht werden, oder mit jenen Flächen, auf denen die landwirtschaftliche Nutzung durch weitere Nutzungen wie Naturschutz, Erholung usw. überlagert wird und nicht zuletzt, wie soll ein Interessenausgleich über den Gesamtraum erfolgen, wenn nicht über das Instrument der Güterzusammenlegung in Kombination mit der Baulandumlegung und evtl. weitern Landumlegungsarten? In diesem Punkt zeigt sich mit aller Deutlichkeit, daß sich die Landwirtschaft nicht aus der Raumplanung herauslösen läßt, sie bleibt mit dieser auf das engste verknüpft. Zugleich zeigt sich darin auch die Bedeutung des Instrumentes der Landumlegung nicht allein in bezug auf die Verbesserung der Agrarstruktur, sondern auch für die Realisierung der Nutzungsordnung und somit zur Entflechtung der Nutzungsinteressen. Die Landumlegung ist daher als Instrument der Raumplanung zur Realisierung der Nutzungsordnung in das Raumplanungsgesetz aufgenommen worden.

Für die Durchführung einer Güterzusammenlegung ist folgerichtig ein ganzheitlicher Ansatz zu wählen, der erlaubt, die Güterzusammenlegung in das raumplanerische Umfeld einzubetten. Systemtechnisch gesprochen bedeutet dies, daß, ausgehend vom System Landwirtschaft, die Raumplanung die Systemumwelt verkörpert (sCHMID und FLURY, 1984). Die Güterzusammenlegung ist so gesehen ein wesentliches Instrument in Kombination mit weiteren Landumlegungsverfahren und Maßnahmen, um räumliche Ordnung vorab im ländlichen Raum zu realisieren. Ein gutes Beispiel für die Entflechtung von landwirtschaftlichen, energiewirtschaftlichen, naturschützerischen, siedlungspolitischen Interessen und der Schaffung einer entsprechenden Raumordnung durch den
Einsatz des Instrumentes der Landumlegung in Kombination mit weiteren Maßnahmen stellt sicher die Reusstalsanierung dar (125 JAHRE ETH ZÜRICH, 1980). Zugleich zeigt die Güterzusammenlegung immer wieder das Spannungsfeld zwischen agrarpolitischen und raumordnungspolitischen Zielsetzungen auf. Erwartungen der Landwirtschaft an die Raumplanung stimmen oft nicht mit den raumplanerischen Zielen überein und umgekehrt oder die raumplanerischen Instrumente genügen den agrarpolitischen Zielsetzungen nicht.

\section{Agrarpolitische Erwartungen an die Raumplanung}

Mit der Aufnahme der landwirtschaftlichen Vorrangflächen in die Richtplanung und der Ausscheidung von Landwirtschaftszonen in der Nutzungsplanung und nötigenfalls der Realisierung dieser Zonenordnung durch die Landumlegung erwartet die Landwirtschaft die Erreichung folgender Ziele:

- Besseren Schutz der landwirtschaftlichen Nutzung und somit einen Beitrag zur Sicherung der Existenz der Bauernbetriebe, indem durch die Landwirtschaftszonen

- eine gesicherte Basis für längerfristige Investitionen (Hochbauten) und Grundlagenverbesserungen (Meliorationen, Erschließungen) geschaffen wird;

- die Verhältnisse für die räumliche Anwendung des $\mathrm{zu}$ revidierenden bäuerlichen Boden- und $\mathrm{Zi}$ vilrechtes geklärt werden;

- eine Beruhigung des landwirtschaftlichen Bodenmarktes erfolgt.

- Erhaltung und langfristige Sicherung genügender Flächen geeigneten Kulturlandes im Hinblick auf die landwirtschaftliche Produktion in Normalzeiten und in Zeiten gestörter Nahrungsmittelzufuhr.

Unbestreitbar konnten im Rahmen der Raumplanung diese Ziele zumindest teilweise erreicht werden, doch ist angesichts der raumplanungsrelevanten Probleme, die sich heute der Landwirtschaft stellen, die Frage offen, ob die raumplanerischen Instrumente und ihre Handhabung genügen, um diese zu bewältigen.

Einige der wesentlichsten, im Zusammenhang mit der Raumplanung stehenden Probleme sollen im folgenden kurz erläutert werden:

\section{a) Bauernhöfe in der Bauzone}

Die heute vielerorts bestehenden Zonenpläne zeigen, daß offenbar aus welchen Gründen auch immer, bei der seinerzeitigen Ausscheidung von Bauzonen keine Rücksicht auf die Bauernbetriebe genommen wurde. So liegen heute eine große Zahl der Bauernhöfe in der Bauzone. Gemäß einer Umfrage des Landwirtschaftsamtes des Kantons Zürich befinden sich ein gutes Drittel aller hauptberuflichen und $50 \%$ aller nebenbe- 
ruflichen Landwirtschaftsbetriebe mit ihren Betriebszentren im Baugebiet (VORSTEHER DES KANTONALEN LANDWIRTSCHAFTSAMTES ZÜRICH, 1981).

Dieses Problem ließe sich einfach lösen, indem diese Betriebe, soweit sie weiterhin lebensfähig sind, mit ihren Betriebszentren an den Dorfrand oder mitten in ihr Bewirtschaftungsgebiet in die Landwirtschaftszone ausgesiedelt würden. Damit wäre nicht nur das Problem mit den Bauernhöfen in der Bauzone gelöst, sondern auch das Horten von Bauland durch die Bauern erschwert, da sie meist Bauland verkaufen müßten, um die neue Siedlung finanzieren zu können. Entgegen der landläufigen Meinung hat sich zum einen im Kanton Zürich gezeigt, daß es nicht die Bauern sind, die vor allem Bauland horten (BIERI, 1983), und zum andern wäre eine Raumplanung, welche die Bauern aus den Dörfern vertriebe, weder landwirtschaftlich noch siedlungs- und gesellschaftspolitisch zu verantworten. Aktive Bauern gehören seit Jahrhunderten zum Dorfbild. Zudem dürfte die Erstellung der notwendigen Gebäude für die Mehrzahl der Bauern an der Finanzierung scheitern. Nach den z. T. negativen Erfahrungen mit Aussiedlungen in der Vergangenheit ist man heute recht zurückhaltend geworden mit dieser Maßnahme.

Verbleibt der Bauernhof aber in der Bauzone, so können ihm je nach Kanton und lokalen Verhältnissen eine ganze Reihe seine Existenz bedrohende Nachteile entstehen:

- Die Betriebsstätte mit Umschwung wird in einen Überbauungsplan- und in Landumlegungsverfahren einbezogen und/oder wird erschließungsbeitragspflichtig. Dies kann unter Umständen zu untragbar hohen Belastungen für den einzelnen Betrieb führen.

- Neu-, Um- und Erweiterungsbauten können erschwert sein.

- Die Vermögenssteuerveranlagung kann bei landwirtschaftlich genutzten Grundstücken in der Bauzone auf der Basis des Verkehrswertes erfolgen.

- Zinsgünstige Investitionskredite oder öffentliche Beiträge können wegen einer höheren Einschätzung des betriebseigenen Landes (unter der Voraussetzung, daß das Hofareal großes Ausmaß hat) gekürzt werden.

- Bewirtschaftungsbeiträge können für landwirtschaftlich genutztes Bauland entfallen.

- Das bäuerliche Erb- und Zivilrecht kann teilweise oder ganz für Bauernhöfe und landwirtschaftlich genutztes Bauland nicht zur Anwendung kommen. Dies wirkt sich insbesondere auf die Veräußerung (Vorkaufsrecht, Einspruchsverfahren, Sperrfrist), die Vererbung und Belastung (Belastungsgrenze für Pfandrechte) aus.

Es bieten sich nun verschiedene Lösungsmöglichkeiten dieses Problemes an. Sicher ist vornehmlich eine raumplanerische Lösung zu suchen. Eine erste solche Lösung könnte darin bestehen, Dorfrandbetriebe der
Bauzone der Landwirtschaftszone zuzuweisen und für die Betriebe im Dorfkern neue Bauernhofzonen zu schaffen. Allerdings benötigt eine solche Lösung immer auch entsprechend flankierende Maßnahmen. Ist ein Bauernhof einmal durch die Siedlungsentwicklung vollständig eingeschlossen worden, so kann seine Situation auch durch Schaffung einer Bauernhofzone nicht verbessert werden. Eine weitere Lösung bestünde darin, die Überbauungsplan-, Landumlegungsund Erschließungsbeiträge zu stunden und die Bauernhöfe mit ihrem Umschwung im Baugebiet in vermögensrechtlicher Hinsicht und in bezug auf das bäuerliche Erb- und Zivilrecht den Bauernhöfen in der Landwirtschaftszone gleichzustellen. Grundsätzlich ist die erste Lösung der zweiten vorzuziehen. Selbstverständlich kann jeder Kanton je nach gegebenen Verhältnissen ähnliche und/oder differenziertere Lösungen treffen; wobei damit auch gesagt ist, daß die entsprechenden Lösungen vorwiegend auf kantonaler Ebene zu suchen sind.

\section{b) Nutzungsüberlagerungen von landwirtschaftlich genutzen Flächen}

Im Gegensatz zur baulichen Nutzung läßt sich die landwirtschaftliche Nutzung durch weitere Nutzungen überlagern.

Solche möglichen Nutzungsüberlagerungen sind:

- der Schutz:

- Gewässerschutz

- Landschaftsschutz

- Naturschutz

- Uferschutz

- Ortsbildschutz (Umgebungsschutz)

- Gefahrenschutz (Lawinen, Wildbäche, Sicherheitsbereiche bei Waffen- und Flugplätzen)

- Erholung im Freien (Wandern, Lagern, Skifabfahrten, Schlittelhänge)

- Kiesabbau und Deponie (zeitlich beschränkte Überlagerung).

Für den Kiesabbau und die Anlage von Deponien sind die Verhältnisse eindeutig. Hier läßt sich von einer Nutzungsüberlagerung sprechen, indem für eine beschränkte Zeit Kies abgebaut resp. Material deponiert wird und vor und nach dieser Zeit das Land landwirtschaftlich genutzt werden kann, unter der Voraussetzung, Kiesabbaustellen und Deponiestandorte werden nach der Ausbeutung resp. Deponie des Materials wieder in einen Zustand gebracht, der eine uneingeschränkte landwirtschaftliche Nutzung erlaubt.

Für den Schutz und die Erholung sind die Verhältnisse schwieriger. Auf der einen Seite beeinträchtig eine extensive Erholung und/oder ein «extensiver» Landschaftsschutz die landwirtschaftliche Nutzung kaum spürbar. Auf der andern Seite kann ein intensiver Schutz oder eine intensive Erholungstätigkeit die landwirtschaftliche Nutzung hinsichtlich ihrer Pro- 
duktivität schwer einschränken. Zwischen beiden Extremen einer kaum beeinträchtigten landwirtschaftlichen Nutzung und einer durch andere Nutzungen dominierten landwirtschaftlichen Nutzung sind alle Abstufungen möglich. Das besondere Problem bei solchen Mehrfachnutzungen liegt einmal darin, abzugrenzen, wann ein Gebiet als Landwirtschaftszone mit explizit oder implizit zugelassenen Nutzungsüberlagerungen oder z. B. als Erholungszone oder Schutzzone mit entsprechender landwirtschaftlicher Nutzung, die im Extremfall eine Landschaftspflege darstellt, zu bezeichnen ist. Zum andern können gerade bei der Freiraumerholung planerische Absichten und Wirklichkeit auseinanderklaffen, da sich der Erholungsdruck auf die Landschaft kaum steuern läßt und vorwiegend eine Funktion der Siedlungsentwicklung darstellt.

Eine besondere Bedeutung hat in diesem Zusammenhang die Überlagerung von Landschaftsschutz- bzw. Naturschutzgebieten mit der landwirtschaftlichen Nutzung. Naturschutz und Landschaftsschutz sind auf die Landwirtschaft angewiesen. Eine naturnahe traditionelle Landwirtschaft wäre sicher auch der wirkungsvollste Landschaftsschutz. Doch der Zwang zur Rationalisierung in der Landwirtschaft hat dazu geführt, daß Landschaftsschutz und landwirtschaftliche Nutzung in vielen Teilen nicht identisch sein können. Landschaftsschutz führt je nach den Verhältnissen zu Einschränkungen in der landwirtschaftlichen Nutzung, und es muß insbesondere hier in Zukunft vermehrt ein Ausgleich zwischen Landwirtschaft und Landschafts- und Naturschutz gefunden werden, bei dem Schutz und Landwirtschaft besser im Einklang stehen.

In diesem Zusammenhang sei nochmals darauf hingewiesen, daß es heute nicht mehr genügt, mittels der Instrumente der Raumplanung den Ort und das $\mathrm{Maß}$ der Nutzungen vorwiegend nach Fläche und ästhetischen Gesichtspunkten festzulegen; es ist für die Nutzungszuweisung zusätzlich das Kriterium der Belastbarkeit der Umwelt miteinzubeziehen.

Ähnlichen Charakter wie die Nutzungsüberlagerung hat für die Landwirtschaftszonen das Bauen außerhalb der Bauzone. Mit der Inkraftsetzung des Raumplanungsgesetzes auf den 1.Jan. 1980 ist die gewässerschutżrechtliche Regelung des Bauens außerhalb der Bauzonen aufgehoben worden. Gemäß Raumplanungsgesetz ist die Voraussetzung für die Erteilung einer Baubewilligung außerhalb der Bauzone die Zonenkonformität oder die Standortgebundenheit. Bauten, die der landwirtschaftlichen Nutzung dienen, sind daher in der Landwirtschaftszone zugelassen, ebenso standortgebundene Bauten wie eine Kläranlage. Für die Bewilligungspraxis spielt daher die Beurteilung, was zonenkonform resp. standortgebunden ist, eine ausschlaggebende Rolle (BEELER, 1984). In diesem Zusammenhang stellt sich auch die Frage, ob bodenunabhängige landwirtschaftliche Produktionsbetriebe wie Geflügelfarmen, Mastbetriebe oder
Erwerbsgartenbau in der Landwirtschaftszone zuzulassen sind, eine Ausnahmebewilligung benötigen oder eher den Bauzonen zuzuordnen sind.

Nutzungsüberlagerungen sowie nicht zonenkonforme, aber standortgebundene Bauten in der Landwirtschaftszone können und sollen nicht verhindert werden. Unter dem Gesichtspunkt der Erhaltung und Sicherung der landwirtschaftlichen Vorrangflächen insbesondere der Fruchtfolgeflächen durch Einführung der Landwirtschaftszone - führen Nutzungsüberlagerungen und die Bewilligung vor allem nicht zonenkonformer Bauten in der Landwirtschaftszone jedoch zu einer Aufweichung der Landwirtschaftszone (4).

Vor diesem Hintergrund ist die heutige Diskussion um eine bessere Sicherung der landwirtschaftlichen Fruchtfolgeflächen zu sehen. Wenn man den Fruchtfolgeflächen schon einen besonderen Schutz zukommen lassen will, so wäre es falsch, nur diese in die Überlegungen miteinzubeziehen. Man müßte diesen Schutz auf die landwirtschaftlichen Vorrangflächen ausdehnen, da nur auf diese Weise die wertvollen und für die Berglandwirtschaft existenzsichernden, notwendigen landwirtschaftlichen Böden der Landwirtschaft uneingeschränkt erhalten bleiben. Die Vorrangflächen im Mittelland sind dann mehr oder weniger identisch mit den Fruchtfolgeflächen.

Wird davon ausgegangen, daß eine einheitliche Landwirtschaftszone nicht genügt, um in genügendem Maße die landwirtschaftlichen Vorrangflächen zu erhalten und zu sichern, so stehen grundsätzlich zwei Lösungsmöglichkeiten zur Diskussion. Einmal ließen sich die landwirtschaftlichen Vorrangflächen in ihrem Bestand über die landwirtschaftliche Gesetzgebung, ähnlich wie dies heute für das Waldareal der Fall ist, schützen. Zum andern könnte die Sicherung über die Raumplanung erfolgen, indem, wie dies z.B. der Kanton Aargau vorsieht, das landwirtschaftliche Gebiet in eine Landwirtschaftszone I und II aufgeteilt wird. Die Landwirtschaftszone I beinhaltet in etwa die Fruchtfolgeflächen bzw. landwirtschaftlichen Vorranggebiete; in der Landwirtschaftszone II sind Nutzungsüberlagerungen und standortgebundene Bauten zulässig. Nachstehende Tabelle 1 gibt über die Vorstellungen des Kantons Aargau Auskunft, welche Bauten und Anlagen in welcher Zone grundsätzlich zulässig resp. nicht zulässig sind, sowie die entsprechenden Musterparagraphen für die Landwirtschaftszone I und II (BACHMANN und SCHMID, 1983)

\section{c) Zusammenwirken zwischen Nutzungsplanung und Strukturverbesserungsmaßnahmen in der Landwirtschaft}

Bei der Durchführung von Güterzusammenlegungen als eine der tiefgreifendsten und wichtigsten Strukturverbesserungsmaßnahmen in der Landwirtschaft entstehen immer wieder offene Fragen in bezug auf die Koordination der Güterzusammenlegung mit der 
Tabelle 1 Zulässige, nicht-zulässige und bedingt zulässige Bauten und Anlagen in den Landwirtschaftszonen I und II

Bauten und Anlagen
- Ökonomiegebäude für den landwirtschaft-
lichen Betrieb wie Stall, Scheune, Remise
u. a. m.
- Rüstplatz, Lagerraum, Lagerplatz,
Verkaufsstelle für die eigene Produktion
aus der Landwirtschaft
- Ökonomiegebäude für den Gemüsebau
und den produzierenden Gartenbau
- Betriebsnotwendiger Wohnraum für den
landwirtschaftlichen Betrieb
- Stöckli
- Bauten für Tierhaltung (Aufzucht, Mast,
Eierproduktion) ohne Betriebsfläche
- Pferdepension/-handel
- Grastrocknungsanlage
- Materialabbau
- Deponien
- Bienenhaus
- Familiengärten
- Ponystall (Hobby)
- Fischzucht
- Kierheime, -klinik, -gehege
- Klubhaus
- Schützenhaus
- Scheibenstand (300 m)
- Flugfeld
- Flugfeld für Modellfliegerei
- Militärische Anlage
- Wasserfassung

Legende $\square$ zulässig $\square$ nicht zulässig $\square$ bedingt zulässig

Ausnahmen regelt Art. 24 RPG und $\S 129$ BauG
Landwirtschaftszone I:

Nutzung:

'Der Landwirtschaftszone I sind Gebiete zugeordnet, an denen ein überwiegendes landwirtschaftliches Interesse besteht. Sie sind der ordentlichen Bewirtschaftung vorbehalten. Zulässig ist die bodenabhängige Produktion in den Bereichen Acker- und Futterbau, der Tierhaltung, Obst- und Rebbau sowie produzierender Gartenbau.

Bauten:

${ }^{2}$ Bauten und Anlagen sind gestattet, soweit sie der zulässigen Nutzung dienen und betriebsnotwendig sind. Bauten und Anlagen, die der bodenunabhängigen landwirtschaftlichen Produktion dienen, sind nur gestattet, soweit sie Zuerwerb zu einem landwirtschaftlichen Betrieb gemäß Abs. 1 darstellen.

\section{Landwirtschaftszone II:}

Nutzung:

${ }^{1}$ Der Landwirtschaftszone II sind Gebiete zugeordnet, an denen ein vorwiegendes landwirtschaftliches Interesse besteht. Sie sind der ordentlichen Bewirtschaftung vorbehalten. Zulässig sind die bodenabhängige und bodenunabhängige Produktion der Landwirtschaft und des produzierenden Gartenbaus.

Bauten:

${ }^{2}$ Bauten und Anlagen sind gestattet, soweit sie der zulässigen Nutzung dienen und betriebsnotwendig sind. Gewächshäuser und andere Bauten der bodenunabhängigen Produktion sind nur an Standorten zugelassen, die landschaftlich tragbar sind.

Ausnahmen in den Landwirtschaftszonen I und II.

Ausnahmen:

${ }^{1}$ Der Gemeinderat kann, mit Zustimmung des kantonalen Baudepartementes, Ausnahmen in den Landwirtschaftszonen I und II bewilligen, wenn der Zweck der Bauten und Anlagen einen Standort außerhalb der Bauzone erfordert und diesen keine überwiegenden Interessen entgegenstehen (Art. 24 Abs. 1 RPG und $\S 129$ Abs. 2 BauG).

${ }^{2}$ Bei der Projektierung und Ausführung von zonenfremden Bauten und Anlagen ist auf die landwirtschaftlichen Interessen Rücksicht zu nehmen.»

Quelle: BACHMANN H. und SCHMID V.: Raumplanung Kanton Aargau, Rechtliche und funktionelle Wirkung der Planungsinstrumente. In: Vermessung, Photogrammetrie, Kulturtechnik Nr. $6 / 83$
Nutzungsplanung. Die Durchführung einer Güterzusammenlegung ist meist ein solch weitreichendes und alle Gemeindestrukturen beeinflussendes Werk, daß sich in der überwiegenden Zahl der Fälle eine Revision der Nutzungsplanung der Gemeinde aufdrängt.
In diesem Zusammenhang ist eine der wiederkehrenden Fragen, wie Landschafts- und Naturschutzgebiete und -objekte zu behandeln sind. Sind diese im Rahmen der Nutzungsplanung endgültig zu bestimmen oder ist diese Aufgabe dem Zusammenlegungs- 
verfahren zu übertragen, indem man sich auf das Verursacherprinzip beruft? Im Grundsatz läßt sich diese Frage wie folgt beantworten (SCHMID w. A., 1984):

Die Güterzusammenlegung ist in erster Linie ein Instrument, das einerseits zur Verbesserung der landwirtschaftlichen Struktur und andererseits zur Umsetzung raumplanerischer Vorhaben dient. Die Naturschutz- und Landschaftsschutzzonen sowie die in Inventaren festgehaltenen Schutzobjekte sind grundsätzlich im Rahmen der Ortsplanung auszuscheiden und durch geeignete Vorkehren im Rahmen der Nutzungsplanung vor Beeinträchtigungen zu schützen. Der Güterzusammenlegung fällt dann die Aufgabe $\mathrm{zu}$, diese Planungen $\mathrm{zu}$ realisieren, indem sie, wenn notwendig, die Schutzgebiete dem Kanton, der Gemeinde oder privaten Natur- und Landschaftsschutzorganisationen zuteilt. Die Güterzusammenlegung gewährleistet damit die Nutzungsordnung. M.E. kann es nicht angehen, auf der instrumentellen Ebene der Güterzusammenlegung aufgrund partikularer Natur- und Heimatschutzinteressen neue Schutzzonen auszuscheiden und diese mit dem Verursacherprinzip zu begründen. Damit würde die Raumplanung der politischen Willensbildung der Bevölkerung entzogen. Selbstverständlich ist damit nicht ausgeschlossen, daß Private, private Organisationen oder das Gemeinwesen Land erwerben und dieses in das Güterzusammenlegungsverfahren einwerfen, mit dem Wunsch, Flächen von besonderer Bedeutung für den Natur- und Landschaftsschutz zugeteilt zu erhalten. Zudem kann oder muß eine Güterzusammenlegung, da sie in alle Lebensbereiche einer Dorfgemeinschaft eingreift, Anlaß zu einer Revision der Ortsplanung sein, im Rahmen derer sich Fragen der Unter-SchutzStellung von durch die Güterzusammenlegung betroffenen Gebieten diskutieren lassen, aber eben, dies im Rahmen der Revision der Ortsplanung. Allerdings kann dort, wo das Güterzusammenlegungsverfahren selbst zu Landschaftsschäden führt, das Verursacherprinzip angewandt werden, indem z. B. die im Zuge des Wegebaus abgeholzten Hecken an entsprechender Stelle neu angelegt werden. Selbstverständlich liegt hier auch ein Ermessensspielraum vor, und die Übergänge sind daher fließend (SCHMID w. A., 1984).

\section{Schlußfolgerungen in bezug auf die Lösung raumplanungsrelevanter Probleme der Landwirtschaft durch die Raumplanung}

Die vorangegangene Diskussion der raumplanungsrelevanten Probleme wie der Bauernhöfe in der Bauzone, der Nutzungsüberlagerung von landwirtschaftlich genutzten Flächen und der Unsicherheiten des Zusammenwirkens von Nutzungsplanung und Strukturverbesserungsmaßnahmen läßt die Frage aufkommen, ob die Raumplanung in bezug auf die raumplanerisch relevanten landwirtschaftlichen Probleme versagt. D.h., sind die Mittel der Raumplanung ungenügend und sind in der Folge die entsprechenden Gesetze und Verordnungen zu ändern, zu ergänzen oder anzupassen, oder reicht die politische Tragfähigkeit nicht aus, um räumliche Konflikte mittels raumplanerischer Mittel lösen zu können?

So wie die räumliche Entwicklung nicht stehen bleibt und die Raumplanung einen dauernden Prozeß darstellt, so ist auch das Raumplanungsrecht der Entwicklung früher oder später anzupassen. Jedoch scheint mir dies in bezug auf die offenen Fragen der Landwirtschaft nicht das dringendste Erfordernis zu sein. Sollten sich zudem Anpassungen und Ergänzungen des Raumplanungsrechtes als notwendig erweisen, so haben diese sicher zunächst auf kantonaler Ebene zu erfolgen.

Hingegen scheint es mir notwendig, daß nach Möglichkeit versucht werden soll, die Mittel der Raumplanung zur Lösung der entsprechenden raumrelevanten landwirtschaftlichen Probleme einzusetzen, so z.B. durch Schaffung von Bauernhofzonen. An dieser Stelle sei allerdings vor einer Atomisierung der Zonenordung gewarnt, indem für jeden Spezialfall eine neue Zone geschaffen wird. Ein solches Vorgehen würde zur Unübersichtlichkeit und einer Gefährdung der großen Linien der Raumplanung führen und zudem eine Rechtsunsicherheit beim Bürger schaffen. D.h., das Raumplanungsgesetz soll besser ausgeschöpft und konsequenter angewandt werden.

Es ist nicht von der Hand zu weisen, daß die geschilderten Probleme auch teilweise darauf zurückzuführen sind, daß der Raumplanungsprozeß oft als reinè planungsrechtliche Aufgabe verstanden wird und man sich zuwenig mit der materiellen Seite der räumlichen Probleme auseinandersetzt. Zudem ist die politische Dimension der Raumplanung in den letzten Jahren eher zurückgegangen.

Neben der notwendigen Weiterentwicklung des Raumplanungsrechtes mit seinen Instrumenten ist $\mathrm{zu}$ hoffen, daß in Zukunft das bestehende Recht konsequenter ausgeschöpft wird und das politische Bewußtsein für räumliche Problemsituationen wächst.

\section{Literatur}

ARBEITSGRUPPE «RAUMPLANUNG UND LANDWIRTSCHAFT DES SCHWEIZERISCHEN BAUERNVERBANDES" (1983): Die Landwirtschaft in der Raumplanung. Schlußbericht. Verlag des Schweiz. Bauernsekretariates, Brugg, S. 23ff.

BACHMANN H., SCHMID V. (1983): Ausscheidung von Landwirtschaftszonen im Kanton Aargau. In: Vermessung, Photogrammetrie, Kulturtechnik Nr. 6/83, Zürich

BEELER U. (1984): Die widerrechtliche Baute. In: Schriftenreihe zur Orts-, Regional- und Landesplanung Nr.32, Institut für Orts-, Regional- und Landesplanung ETH Zürich

BIERI H. (1983): Bauernhöfe in den Bauzonen - Erfahrungen im Kanton Zürich. In: Vermessung, Photogrammetrie, Kulturtechnik Nr. 8/83, S. 281ff., Zürich 
125 JAHRE ETH ZÜRICH (1980): Die Aargauische Reusstalsanierung und die Forschung der ETH Zürich. In: Schweizer Ingenieur und Architekt Nr. 14/80, 98 Jrg., Zürich

EWALD K.C. (1978): Der Landschaftswandel, Zur Veränderung Schweizerischer Kulturlandschaften im 20 Jhdt. In: Sonderdruck aus: Tätigkeitsberichte der Naturforschenden Gesellschaft Baselland, Band 30, S. 55-308, Liestal

HÄBERLI K. und STALDER K. (1979): Entwicklung der landwirtschaftlichen Nutzflächen in der Schweiz, 1939-1975, aufgrund der eidgenössischen Betriebszählungen. In: Raumplanung Schweiz Nr. 2/79, S. 3ff. (3-12 u. 21-27), EJPD, Bern

KAULE G. (1980): Umweltverträglichkeitsprüfung in der räumlichen Planung. In: DISP Nr. 59/60, Dokumente und Informationen zur Schweizerischen Orts-, Regional- und Landesplanung, S. 34, Zürich

LENDI M. (1984): Gesamtplanung und Sachplanungen. In Recht und Politik der Raumplanung. Schriftenreihe zur OrtsRegional- und Landesplanung Nr. 31, ORL-Institut ETH Zürich, S. $31 \mathrm{ff}$

SCHMID W. A., FLURY A. (1984): Systemtechnisches Vorgehen in der Güterzusammenlegung erläutert am Beispiel OtelfingenBoppelsen. In: Verlag der Fachvereine Zürich

SCHMID W.A. (1984): Meliorationen im Spannungsfeld verschiedener Interessen aus der Sicht der Methodik. In: Vermessung, Photogrammetrie, Kulturtechnik Nr. 7/84, Zürich, S. 228ff.

VORSTEHER DES KANTONALEN LANDWIRTSCHAFTSAMTES ZÜRICH (1981): Erhebung über die Ackerbaustellenleiter im Winter 1980/81 auf Anregung der SVIL

\section{Anmerkungen}

(1) Zur Beschreibung der Landschaft mittels Naturpotentialen. Vgl. Hase G. (1978): Zur Ableitung von Naturraumpotentialen. In: Petermanns Geogr. Mitteilungen, H. 2. S. 113-125.

(2) Die Art.22ter BV die Eigentumsgarantie betreffend und Art. 22quater BV die Raumplanung betreffend, sind zunächst zwei voneinander unabhängige Artikel. Sie wurden aber nicht von ungefähr gleichzeitig zur Abstimmung gebracht. Es bestand damit die Absicht, das Bodenrecht neu zu ordnen, Sieht man beide Artikel im Zusammenhang, so ergibt sich insofern eine materielle Einheit, indem damit ausgesagt wird, $\mathrm{da}$ ß die Raumplanung im öffentlichen Interesse liegt und $\mathrm{zu}$ Beschränkungen des Eigentums führen kann und darf.

(3) Der Fachbereich Landschaft am ORL-Institut der ETHZ beschäftigt sich seit geraumer Zeit mit der Frage der Integration ökologischer Forderungen in die Raumplanung. Vgl. dazu: Gfeller et al. (1984): Berücksichtigung ökologischer Forderungen in der Raumplanung. In: Berichte zur Orts-, Regional- und Landesplanung Nr. 46, ORL-Institut ETH Zürich, 1984.

(4) In einem Artikel in der Neuen Zürcher Zeitung vom Freitag, den 13. Juli 1984, Nr.161, wendet sich der Bund gegen die zu laxe Praxis der Bewilligung von Bauten außerhalb der Bauzone. Er führt aus, daß im Jahr 1983 rund 11000 Gesuche von den Kantonen zu behandeln waren, $10 \%$ mehr als 1982. Davon wurden gegen $86 \%$ bewilligt, wovon $51 \%$ nicht zonenkonforme und somit standortbedingte Bauten. Das Bundesamt für Raumplanung interpretiert diese Bewilligungspraxis aufgrund dieses Artikels als eine Durchlöcherung des Raumplanungsprinzipes. 